\title{
Deformation in cooperative molecular motors
}

\author{
S. Taneri ${ }^{a}$ and M. Cemal Yalabık \\ Department of Physics, Bilkent University, 06533, Bilkent, Ankara, Turkey
}

Received 20 February 2001 and Received in final form 31 May 2001

\begin{abstract}
We implement a model to represent the effect of the deformation of the backbone of a system of motor proteins while sliding on a track filament. This model incorporates a nearest neighbor interaction term among the motors for the deformation energy. Correlations induced by this term result in increased motor force for inter-particle distances small compared to the ratchet period.
\end{abstract}

PACS. 87.15.-v Biomolecules: structure and physical properties - 87.15.Aa Theory and modeling; computer simulation - 87.16.Nn Motor proteins

\section{Introduction}

The complex phenomenon of muscle contraction can be modeled in simple terms via the investigation of the subject molecular motors. These molecular motors, acting cooperatively yield the motion which governs the muscle contraction phenomenon in some living organisms. In this respect, cooperative molecular motors have been subject of interest and the relation between several dynamic quantities such as force-velocity relations have been investigated [1-6]. These systems may be modeled as in relative motion with respect to a track along micro-tubules. The multi-motor filament moves along these micro-tubules that has a ratchet potential built into it. It is interesting that directed motion comes along with the introduction of this "ratchet" potential even when it is spatially symmetric [7]. In the presence of a symmetric saw-tooth potential, the directed motion can still be maintained via the Adenosinetriphosphate (ATP) regions on the track. The function of these regions is to break the detailed balance and thus create a dynamical instability. This leads to spontaneous symmetry breaking due to the asymmetric motor protein distribution on the track filament and results in sustained motion. Several generalizations of the original models $[1,4,7]$ such as those incorporating an elastic coupling of motor molecules to a rigid backbone and elastic coupling to the environment have been proposed for study [1]. Elastic coupling to the environment has been modeled by the connection of the rigid filament backbone to the outside environment via a spring-like force. This model has been used to explain the oscillations occurring in the muscle of some insects [7]. Deformation phenomenon stems from internally generated forces which so far has been examined as axonemal deformations [8-10].
The elastic coupling of the motors to the backbone mentioned above, has been studied separately, which in fact is also related to the generation of internal forces. On the other hand, the attachment of the motors to a rigid backbone via springs can be used to study the effects of the elasticity of the motor material itself. The effect of distance variations between motors on the force-velocity relations was studied in references $[2,3]$. The deformation was described in terms of a harmonic potential with and without strain-dependent detachment rate.

In this present work, we study the effects of the deformation of the backbone material with motors particles connected to this backbone. The energetics of the deformation is included through an Ising-like model as will be described below. The backbone then may be assumed to be composed of motors which can be in one of the energy levels of the well-known two state system $[1,4,7]$. The energetics of the deformation enhance the the correlations in the states of neighboring particles, which in turn influences the collective motor properties.

\section{The model}

The model is based on a triangular symmetric saw-tooth potential for the track filament of the motor and a higher constant potential for the backbone (Fig. 1). Here, $E_{1}(x)$ and $E_{2}$ are the ratchet potential [11] and the constant potential respectively. The energy of the system is given by:

$$
H=\sum_{i} \frac{1-S_{i}}{2} E_{1}\left(x_{i}\right)+\sum_{i} \frac{1+S_{i}}{2} E_{2}-\frac{\epsilon}{4} \sum_{\langle i j\rangle} S_{i} \cdot S_{j}
$$

\footnotetext{
a e-mail: taneri@fen.bilkent.edu.tr
} 


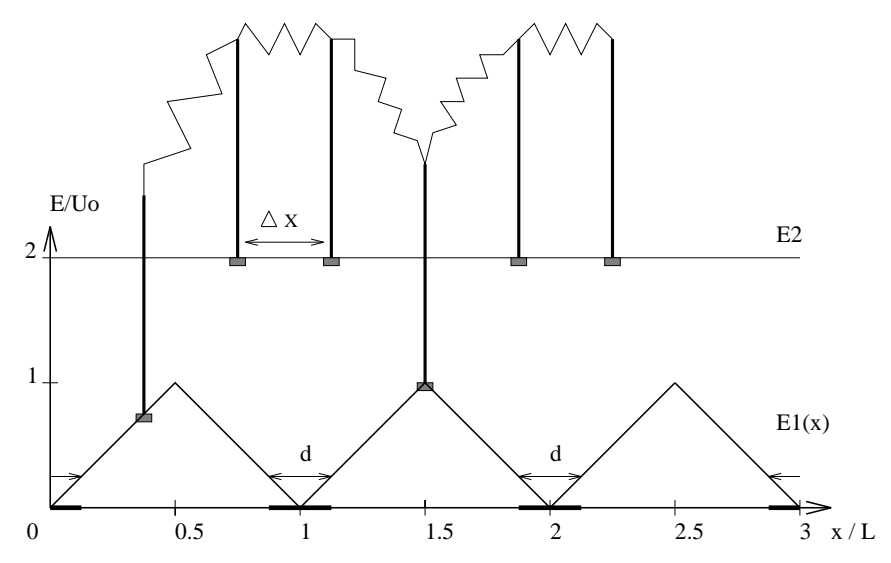

Fig. 1. A diagram of the $E_{1}(x)$ and $E_{2}$ potentials. The width of the region where ATP excitation occurs is $d=0.1$ and $U_{0}$ is the height of the ratchet potential.

where $\epsilon / 4$ is a positive constant which denotes the amplitude of the potential representing the deformation energy. $S_{i}$ indicates the state of the $i$ th particle with $S_{i}=1$ for the detached and $S_{i}=-1$ for the attached states and the sum over $\langle i j\rangle$ indicates a summation over all nearest neighbor pairs. One should note that the deformation energy is increased by an amount $\epsilon / 2$ when the adjacent particles are in different states (springs stretched) in comparison to the case when they are in the same state (springs not stretched).

In our generalization of the model, the major effect of the deformation is assumed to be related to the states of the particles (i.e. attached or detached). Admittedly, a deformation might correspond to a displacement of the particles relative one another in the direction of the motor motion. In this work, we are considering a limit in which such relative displacement results in small changes in energy and hence in the transition rates in comparison to the changes resulting from being in attached or detached states.

We carry out a Monte Carlo simulation of this system using the dynamics given by the transition rates $[4,7,5]$ :

$$
\begin{array}{r}
\omega_{1}(i)=\omega_{2} \exp \left[\left(\epsilon \frac{S_{i+1}+S_{i-1}}{2}+E_{1}\left(x_{i}\right)-E_{2}\right) / k T\right] \\
+\Omega\left(x_{i}\right)
\end{array}
$$

where $\omega_{1}(i)$ is the transition rate for the $i$ th particle from the attached state to the detached state. The transition rate for the reverse process $\omega_{2}$ is chosen as 1 and defines the time scale. Note that $\Omega\left(x_{i}\right)$ destroys the detailed balance and represents the ATP excitations [4]. This effect is assumed to be present at certain regions on the track, as shown in Figure 1. We will call $\Omega$ as "the excitation amplitude" for consistency with previous work [4]. We take $\Omega=10$ a value sufficiently large compared to the time scale defined by $\omega_{2}$. Note that, $k$ and $T$ are the Boltzmann constant and temperature respectively. The functioning of the motor relies on the release of energy from the particles to one side of the potential ramp, while they are being detached from the other side by the excitation. Motion of a motor makes it more probable to release its energy on the side of the potential ramp which enhances the motion in that direction. This leads to a dynamic instability and hence a spontaneous velocity. The correlations generated by the deformation energetics result in more of the excited motors reaching the "other side of the ramp", which yields a better collective motor performance for some range of parameters. On the other hand, these correlations make an analytical analysis of this system prohibitively complicated.

\section{Calculation}

The numerical computation was started by assigning the motor an initial velocity. The motor is assumed to consist of 1000 particles separated by an amount $\Delta x$ on a ratchet potential with spatial period $L$. The force acting on the protein was then calculated at each time step. The total collective motor force $\left(F_{\text {mot }}\right)$ due to potentials indicated in Figure 1 is determined by adding up the forces for both attached and detached particles (the force on a detached particle is trivially zero whereas the force on an attached particle is negative the slope of the model symmetric sawtooth potential). The protein is moved at the initial constant velocity, and the force acting on the individual motors is averaged over. The inter-particle distance $\Delta x$ is irrationally related to $L$ so that the distribution of motors is incommensurate with $L$. This guarantees that for a sufficiently long filament the motors will be distributed uniformly over the potential period and therefore the force will be constant. The state of the motors was changed at each time step by the following procedure: the total probability of any transition in a time interval $\Delta t$ is calculated

$$
P=\Delta t \sum_{i=1}^{n} \omega(i)
$$

where $\omega(i)$ is the transition rate of the $i$ th motor in the system $\left(\omega(i)\right.$ is either $\omega_{1}(i)$ or $\omega_{2}$ depending on the state of the $i$ th motor). The time step $\Delta t$ is chosen such that the total probability $P$ is much less than 1 so that the probability of more than one transition in $\Delta t$ is negligibly small. In order to comply with this constraint $F_{\text {mot }}$ is calculated through averaging $10^{5}$ time steps of $\Delta t=10^{-3}$ after the system is sufficiently relaxed, except forlarge values of $\epsilon$ where averaging is done through $10^{6}$ time steps of $\Delta t=10^{-4}$. Whether or not a transition will take place is decided at each time step with probability $P$. The particular state which will go through the transition is chosen randomly with a probability proportional to its transition rate. In order to use unitless quantities, we scale the lengths by the period of the ratchet potential $L$, the energies by the magnitude of the symmetric saw-tooth potential $U_{0}$ and the masses by the mass of a particle $M$. 


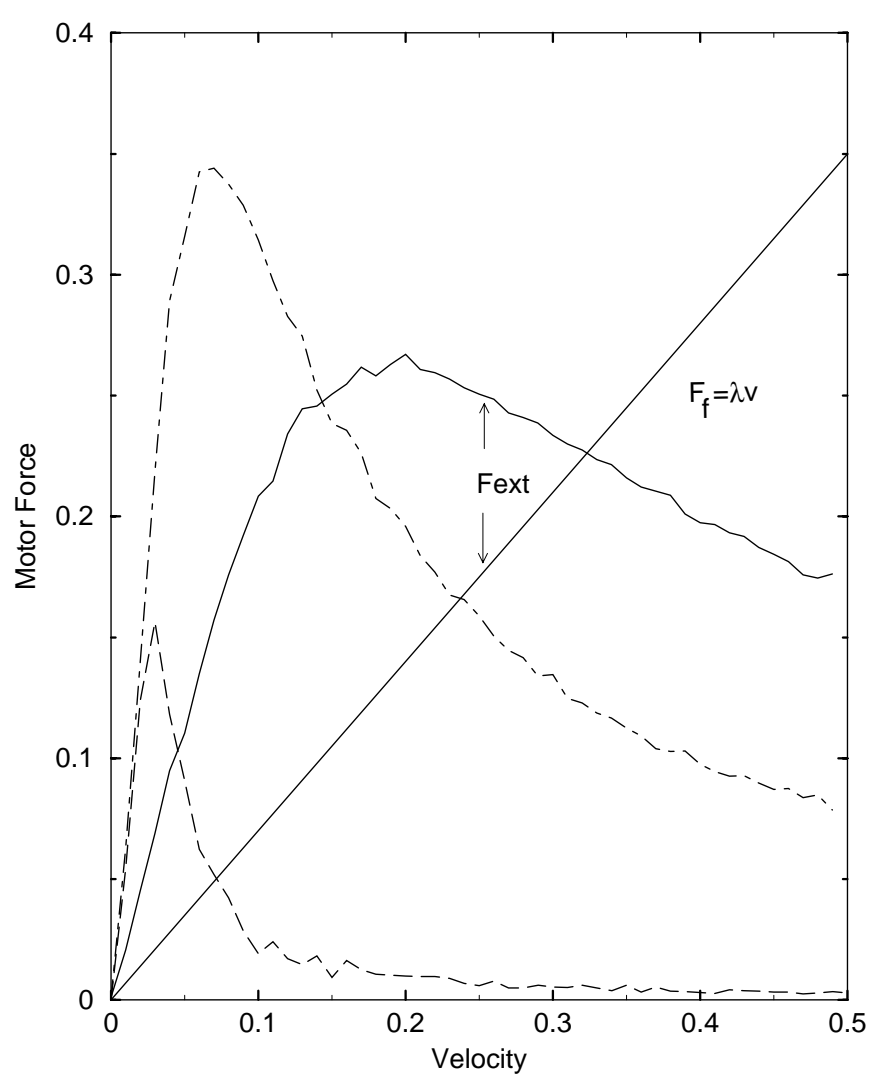

Fig. 2. The velocity dependence of the $F_{\text {mot }}$ for $\epsilon=0$ (solid), $\epsilon=4$ (dot-dashed), $\epsilon=8$ (long dashed) for inter-particle distance $\Delta x=\sqrt{2} / 16$.

The time variable is then scaled by $\sqrt{M L^{2} / U_{0}}$. We have chosen the energy of the detached state as $E_{2} / U_{0}=2$, and $k T / U_{0}=1$. The inter-particle distance $\Delta x$ is chosen as $\sqrt{2} / 4$ which is typically around 0.24 for kinesin given that the motor density is $5 \times 10^{8} \mathrm{~m}^{-1}$ [8] and potential period is $8.2 \mathrm{~nm}$ for kinesin [13].

The force developed by the motors as a function of $v$ for different values of $\epsilon$ is shown in Figure 2. For intermediate values of $\epsilon$, the introduction of correlations results in enhancement of attached and detached particle domains in accordance with the states the adjacent particles are in. For small values of $\Delta x$, these adjacent motors are more likely to be on the same ramp. For very large values of $\epsilon$ however, ATP excited adjacent motors tend to "stick" to their excited states so that the mechanism generating the motor force is now blocked. It should be remembered that this force is balanced by two other forces, a "friction force", which is usually taken to be proportional to the velocity $F_{\mathrm{f}}=-\lambda v$ and an "external force" $F_{\text {ext }}$, which may be present due to possible external effects. The friction force may be trivially subtracted from our results in Figure 2 to obtain the external motor loading as a function of velocity in the form $F_{\text {ext }}=F_{\text {mot }}-\lambda v$. As can be seen from Figure 2, the friction coefficient $\lambda$ cannot be larger than a critical value $\lambda_{\mathrm{c}}$ for any particular value of $\epsilon$, if the

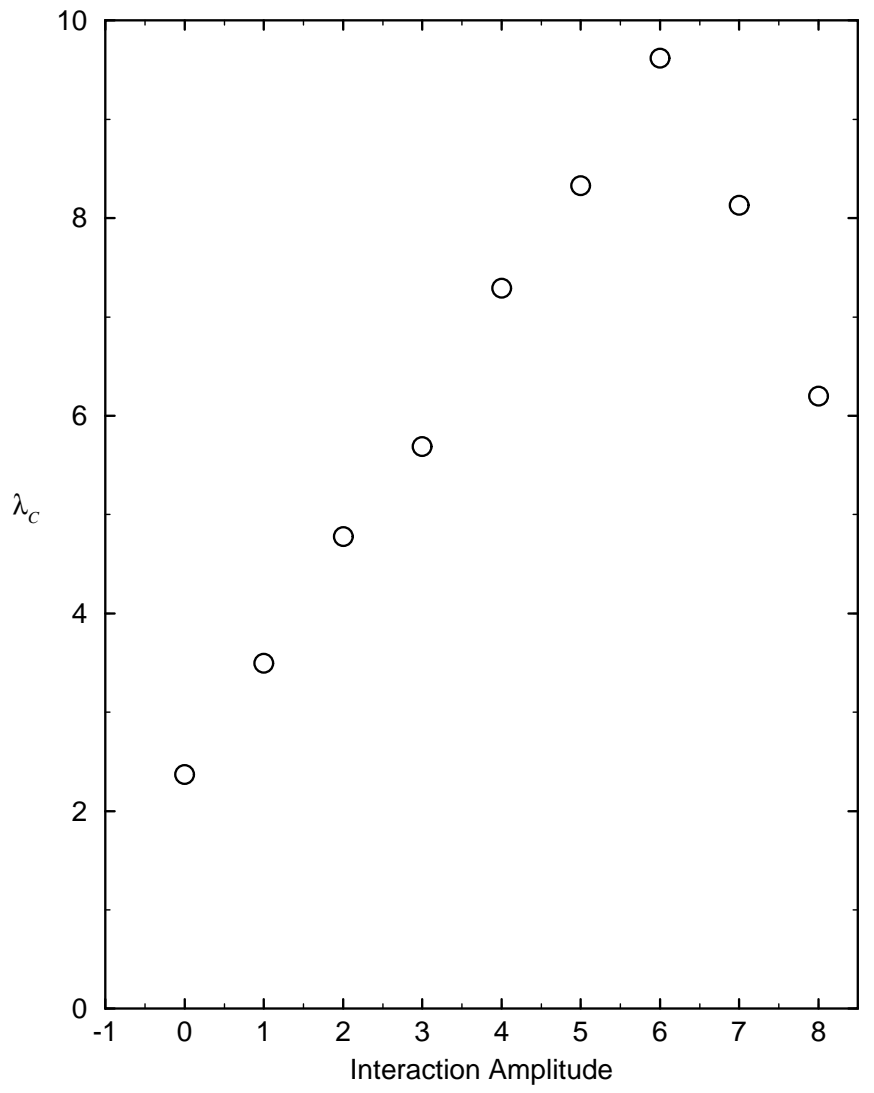

Fig. 3. The critical value of $\lambda, \lambda_{\mathrm{c}}$ versus the interaction amplitude $\epsilon$ for $\Delta x=\sqrt{2} / 16$.

system is to function as a motor (i.e. $F_{\text {ext }} \geq 0$ ) [4]. That is, in order to get a non-zero stable solution, $\lambda$ will have to be smaller than $\lambda_{\mathrm{c}}$, which is the slope of the tangent to the $F_{\text {mot }}$ versus velocity curve at the origin. These critical values of $\lambda$ are displayed as a function of $\epsilon$ in Figure 3.

\section{Discussion}

According to Figures 2 and 3, we see that "maximum collective motor force" $F_{\max }$, and $\lambda_{c}$ increase with the increase of $\epsilon$. But, for larger values of $\epsilon$ we see a drop in both $F_{\max }$ and $\lambda_{\mathrm{c}}$ since the immense increase in the correlation spurs attached particles to the upper constant energy level which inhibits force generation as was mentioned previously. The decrease in velocity at $F_{\max }$ as correlation increases is not surprising since the increase in $\epsilon$ enables the particles to remain in their excited states for a longer time. This energy can then be supplied to the motor especially if it travels with a lower velocity.

The inter-particle distance dependence of $F_{\max }$, for different $\epsilon$ values is displayed in Figure 4. For non-zero $\epsilon$ and small values of $\Delta x$, the inter-particle distance, $F_{\max }$ increases, while for the values of $\Delta x$ around half the spatial period $F_{\max }$ decreases. This is in contrast to the $\epsilon=0$ 


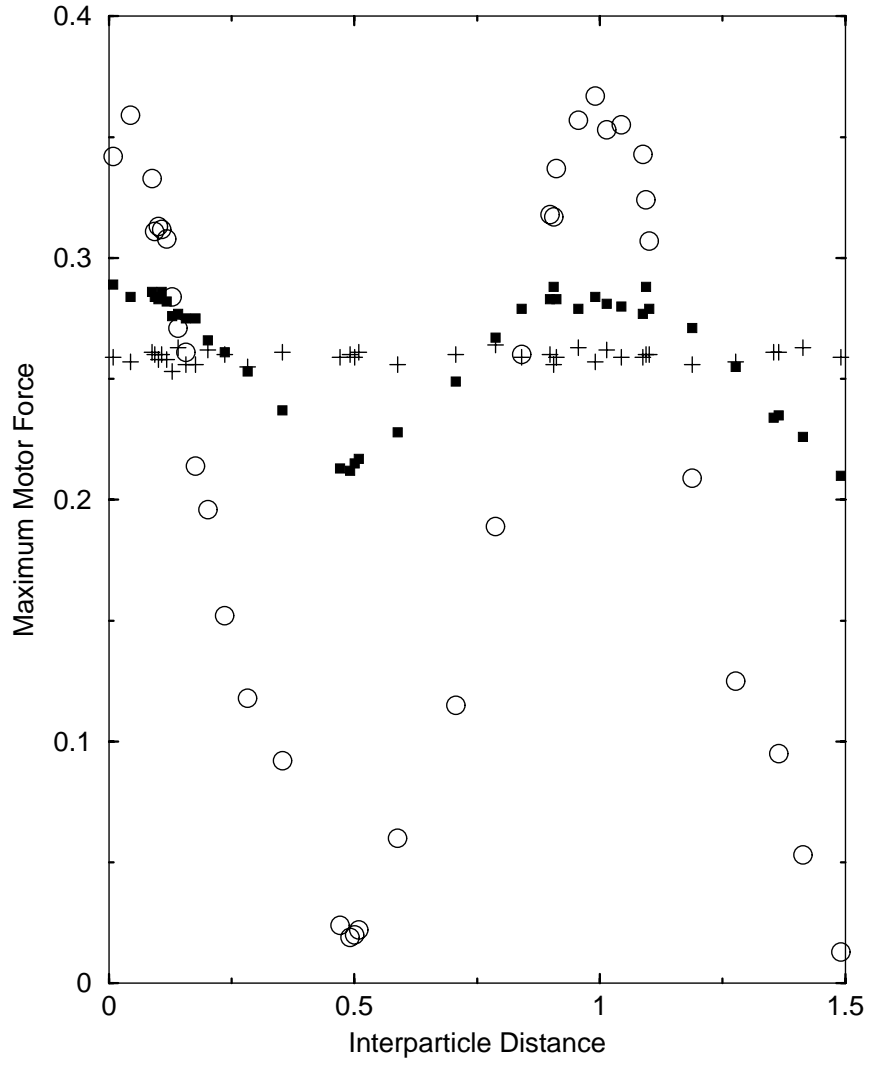

Fig. 4. The inter-particle distance dependence of $F_{\max }$ for $\epsilon=4$ (circle), $\epsilon=1$ (small filled-square) and $\epsilon=0$ (plus). We have extended the plot beyond the expected period of $\Delta x=1$, as a check of our computation.

case where $F_{\max }$ stays constant as expected because, the particle distribution is uniform and incommensurate in the absence of correlations. The size of the fluctuations in Figure 4 gives an idea about the magnitude of the systematic errors due to finite length of our system.

The population of the particles in the attached states is shown in Figure 5. It is seen that the population pattern is deformed for larger inter-particle distances. To understand the multiple peaked structure of the $\Delta x=\sqrt{2} / 4$ graph, consider the problem of arranging three adjacent particles to obtain a maximum amount of force. For this interparticle distance one cannot have all three adjacent particles attached to the symmetric saw-tooth potential so that all three experience a positive force. This explains why $F_{\max }$ decreases for values of $\Delta x$ around half the spatial period in Figure 4 . At the same time, $F_{\max }$ increases for small values of $\Delta x$ in Figure 4 , since one can have all three adjacent particles experiencing a positive force due to the symmetric saw-tooth potential. Now, for interparticles distance $\Delta x=\sqrt{2} / 4$, there are three intervals for the central particle in which two of the three particles experience a positive force while avoiding the ATP present regions [14]. We expect to see a population growth in these intervals so that the motor attains $F_{\max }$. Indeed, Figure 6

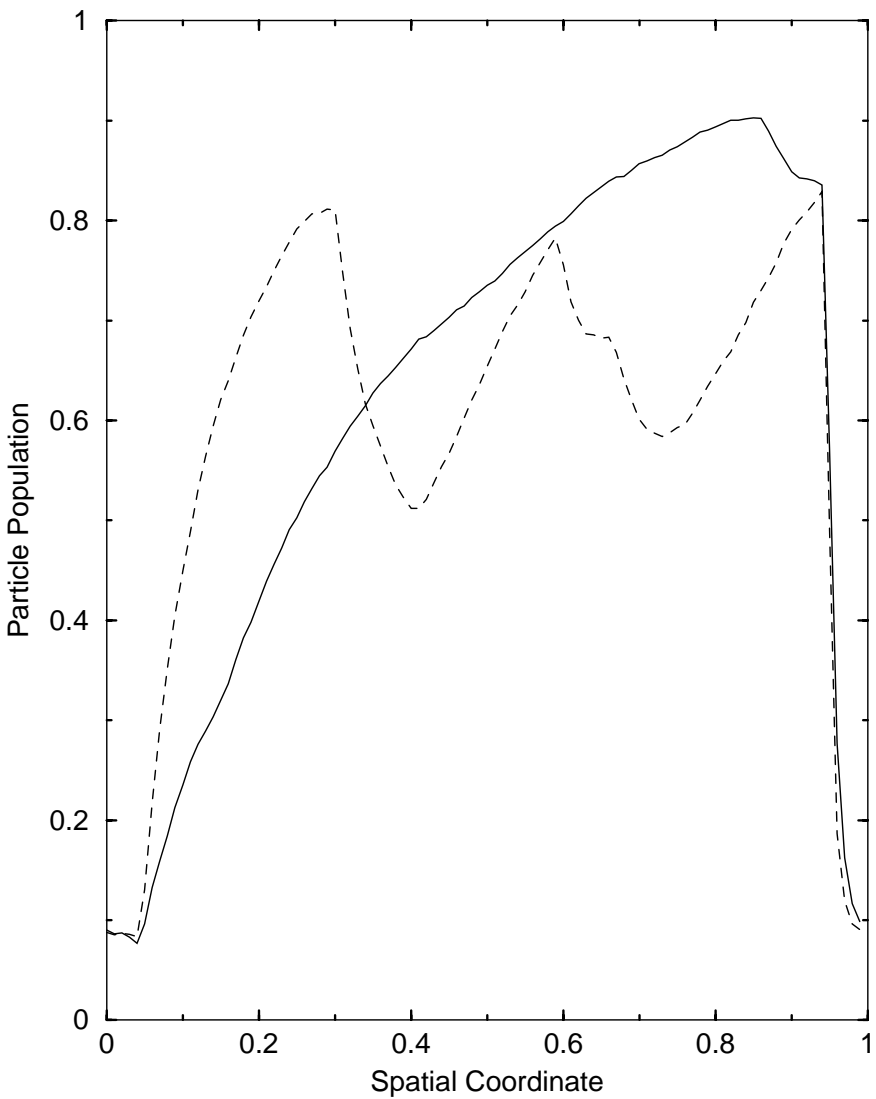

Fig. 5. Population versus spatial coordinate of particles attached to the ratchet potential at maximum motor force and for inter-particle distances $\Delta x=\sqrt{2} / 16$ (solid) and $\sqrt{2} / 4$ (dashed) with $\epsilon=4$.

shows how this results in three peaks which are responsible for the deformation shown in Figure 5. One can also see that the overwhelming contribution to the total force comes from triplets of neighboring motors all in attached states.

The $\epsilon$ dependence of $F_{\max }$ is studied in Figure 7 which simply summarizes the dynamics of the system examined earlier. The error bars in Figure 7 have been determined through a somewhat optimistic estimate, dividing the mean square fluctuations of the measured quantity by the square root of the number of independent samples in the average. This number has in turn been estimated as the total simulation time divided by $3 / \Omega$, which is one of the time scales in the system. In particular, systematic errors which may be the result of the finite size of the system are expected to be small and not included in this analysis.

We have also looked at the efficiency of the process, as defined by the ratio of power delivered by the collective motor to the total power supplied by the ATP excitation [1]. Consistent with our other results we find that the 


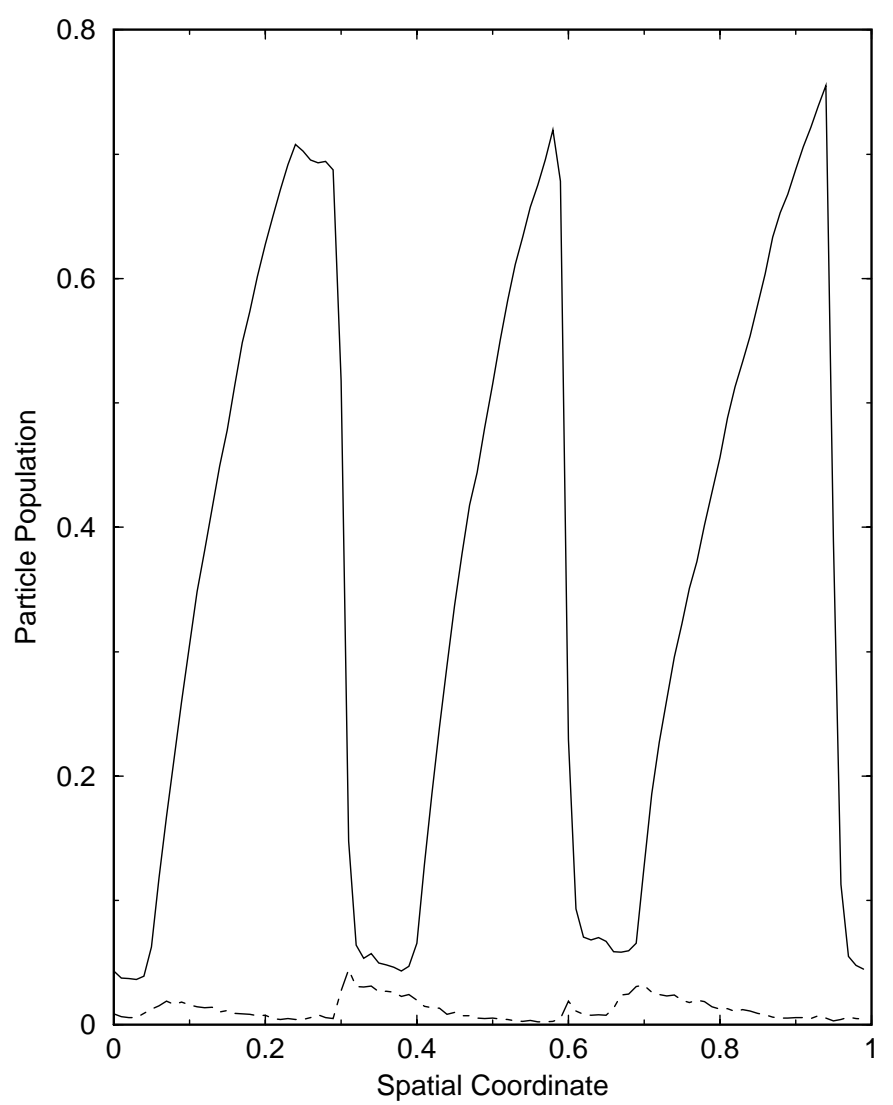

Fig. 6. Population versus spatial coordinate of a central particle of a triplet of neighboring particles all attached to the ratchet potential (solid), and with the central particle attached while both of the neighbors detached (dot-dashed) at maximum motor force. Inter-particle distance $\Delta x=\sqrt{2} / 4$ and $\epsilon=4$.

efficiency of the finite $\epsilon$ collective motor is larger than that for $\epsilon=0$ at smaller values of the velocity.

We conclude that deformation energy brings significant modifications and a richer structure and may yield a "better" motor for some range of parameters.

MCY acknowledges research support from Turkish Academy of Sciences.

\section{References}

1. Force and Motion Generation of Molecular Motors: A Generic Description, F. Jülicher in Transport and Structure: Their Competitive Roles in Biophysics and Chemistry, edited by S.C. Müller, J. Parisi, W. Zimmermann, Lect. Notes Phys. (Springer, 1999).

2. A. Vilfan, E. Frey, F. Schwabl, Europhys. Lett. 45, 283 (1999).

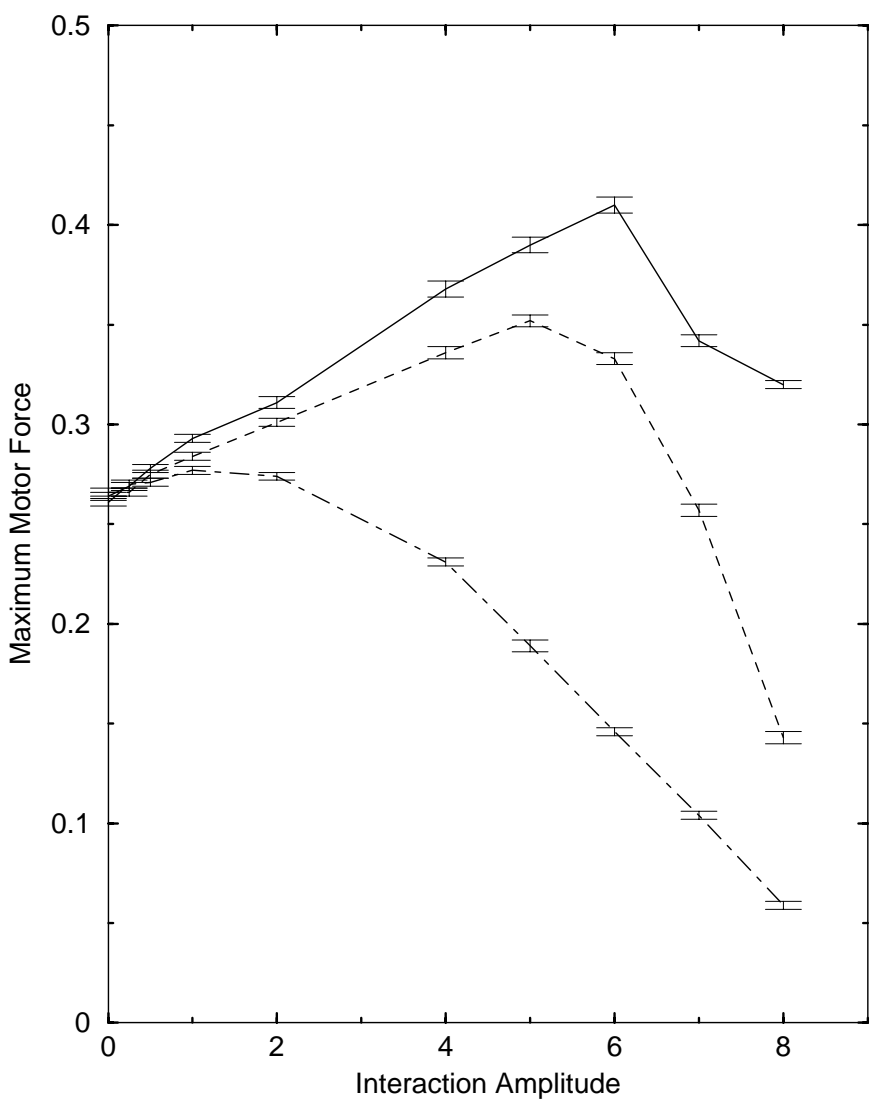

Fig. 7. $F_{\max }$ versus interaction amplitude $\epsilon$ for the three different inter-particle distances $\Delta x=\sqrt{2} / 32$ (solid), $\sqrt{2} / 16$ (dashed), and $\sqrt{2} / 8$ (dot-dashed).

3. A. Vilfan, E. Frey, F. Schwabl, Eur. Phys. J. B 3, 535 (1998).

4. F. Jülicher, J. Prost, Phys. Rev. Lett. 75, 2618 (1995).

5. J. Prost, J.-F. Chauwin, L. Peliti, A. Ajdari, Phys. Rev. Lett. 72, 2652 (1994).

6. R. Dean Astumian, M. Bier, Phys. Rev. Lett. 72, 1766 (1994).

7. F. Jülicher, J. Prost, Phys. Rev. Lett. 78, 4510 (1997).

8. S. Camalet, F. Jülicher, New J. Phys. 2, 24 (2000), physics/000310130 Mar 2000.

9. S. Camalet, F. Jülicher, J. Prost, Phys. Rev. Lett. 82, 1590 (1999).

10. R. Everaers, F. Jülicher, A. Ajdari, A.C. Maggs, Phys. Rev. Lett. 82, 3717 (1999).

11. T.E. Dialynas, K. Lindenberg, G.P. Tsironis, Phys. Rev. E 56, 3976 (1997).

12. J.H. Li, Z.Q. Huang, Phys. Rev. E 57, 3917 (1998).

13. M.E. Fisher, A.B. Kolomeisky, Physica A 274, 241 (1999).

14. These intervals are $[0.15,0.30],[0.50,0.60]$ and $[0.85,0.95]$. 